\title{
expérience in situ et modélisation du comportement des cavités salines utilisées pour le stockage de gaz
}

\author{
par \\ P. Boucly $\left({ }^{*}\right)$ \\ Ingénieur/Département "Réservoirs Souterrains » \\ Direction des Études et Techniques Nouvelles, Gaz de France
}

\section{Suivi des cavités salines}

Compte tenu des profondeurs d'implantation des cavités de Tersanne [2] et d'Etrez [8] et des mesures des caractéristiques mécaniques effectuées au laboratoire sur des carottes de sel, le massif salifère dans lequel sont creusées les cavités est le siège de déformations permanentes : il en résulte une variation de volume des cavités. C'est pourquoi dès le début du projet de stockage à Tersanne, des moyens de surveillance ont été mis au point afin d'assurer une surveillance régulière des cavités salines.

\subsection{Thermométries}

\subsubsection{Principe de la méthode}

Afin de connaître le volume occupé par une masse de gaz, l'idée la plus naturelle qui vient à l'esprit est I'utilisation de l'équation d'état [4] :

$$
\frac{\mathrm{pV}}{\mathrm{zT}}=\frac{\mathrm{p}_{0}}{\mathrm{~T}_{0}} \cdot \mathrm{s}
$$

$\begin{aligned} \text { oủ } \mathrm{p} & =\text { pression moyenne en cavité, } \\ \mathrm{V} & =\text { volume occupé par le gaz, } \\ \mathrm{T} & =\text { température moyenne en cavité, } \\ \mathrm{z} & =\text { facteur de compressibilité du gaz à la } \\ & \text { pression p et à la température } \mathrm{T}, \\ \mathrm{S} & =\text { stock exprimé dans les conditions normales }\end{aligned}$ de température $T_{0}$ et de pression $p_{0}$.

II suffit donc de connaître les variables d'état du gaz ainsi que le stock pour en déterminer le volume. Or. compte tenu du mode de complétion adopté (production du gaz par annulaire $7^{\prime \prime}-4^{\prime \prime}$, tubing central $4^{\prime \prime}$ plein d'eau), les seules grandeurs accessibles à la mesure

(*) Texte de la communication présentée le 17 octobre 1980 au Comité Français de Mécanique des Roches. directe sont la pression en tête de puits et le profil de température sur toute la hauteur du puits (y compris la cavité) par enregistrement d'une diagraphie thermométrique continue.

Grâce à un algorithme d'intégration numérique, il est possible, connaissant la pression en tête de puits et le profil continu de température dans le puits, de calculer la pression au fond (en tenant compte éventuellement des pertes de charges dans le cas où la cavité est en débit).

Un des problèmes majeurs de cette technique d'évaluation du volume libre d'une cavité en recourant à l'équation d'état, est celui de l'incertitude grevant cette estimation; en effet l'incertitude est donnée par :

$$
\frac{\Delta V}{V}=\frac{\Delta S}{S}+\frac{\Delta P}{P}+\frac{\Delta T}{T}+\frac{\Delta Z}{Z}
$$

Compte tenu des appareils de mesure utilisés, la somme des trois derniers termes $\left(\frac{\Delta P}{P}+\frac{\Delta T}{T}+\frac{\Delta Z}{Z}\right)$ est de l'ordre de $2 \%$.

Par contre l'incertitude sur les stocks - mesures sur les rampes de comptage - est plus délicate à estimer. Bien que très pénalisante, l'approche la plus simple consiste à admettre que l'incertitude sur une variation monotone de stock $\frac{\Delta \delta S}{\delta S}$ est constante.

Le stock $S$ en place est la somme de mouvements de gaz élémentaires :

$$
\begin{array}{cc}
S=\Sigma \delta S_{i} \\
\text { d'où : } \quad \Delta S=\Sigma \Delta \delta S_{i}=\frac{\Delta \delta S}{\delta S} \Sigma\left|\delta S_{i}\right| \\
\text { et : } \quad \frac{\Delta S}{S}=\frac{\Delta \delta S}{\delta S} \times \frac{\Sigma\left|\delta S_{i}\right|}{\Sigma \delta S_{i}} .
\end{array}
$$

On voit donc que cette incertitude ne peut que croître avec le temps. Après quelques années d'exploitation, le terme d'incertitude concernant les comptages devient 

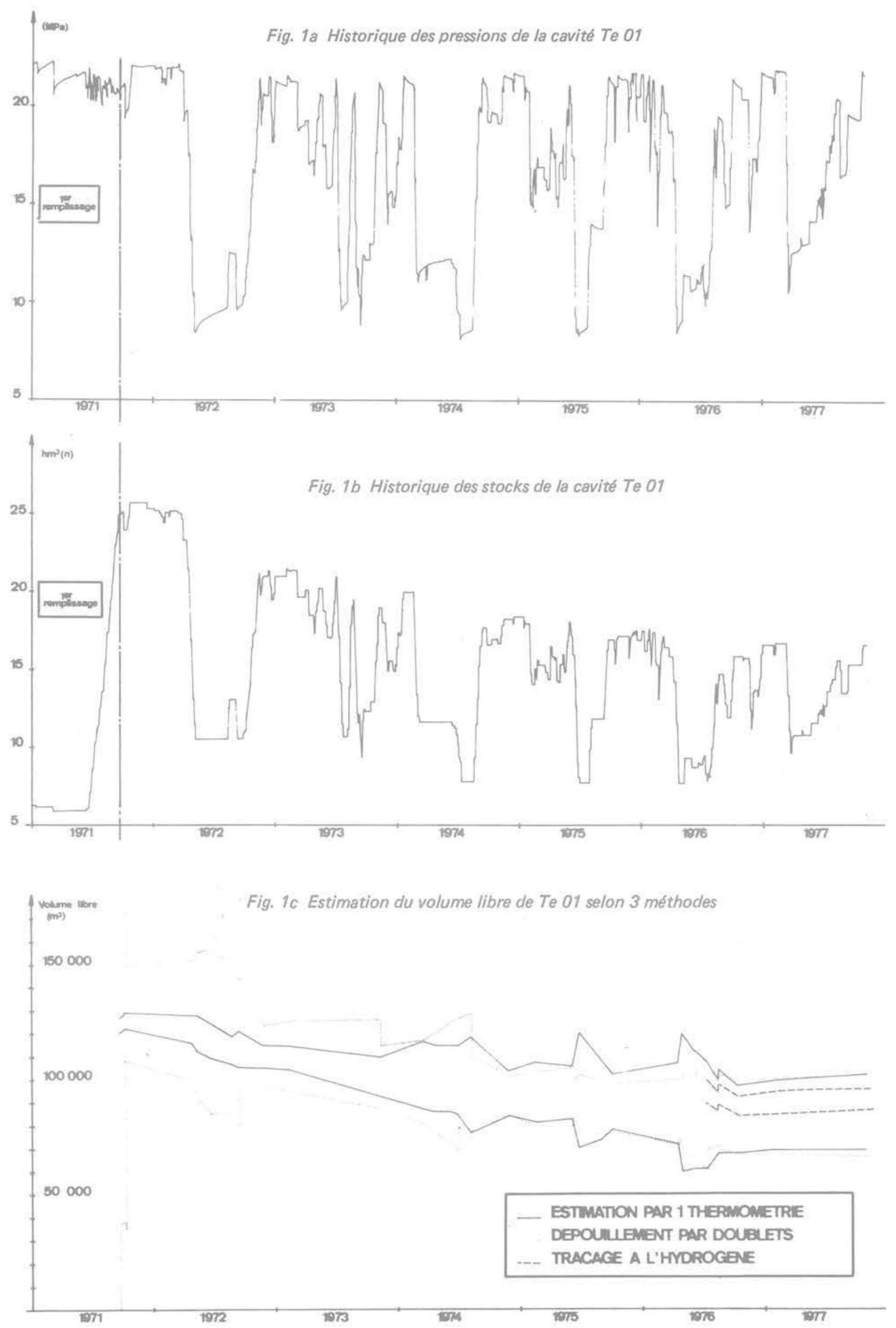
dominant par rapport aux autres incertitudes et rend peu significatives les déterminations de volume ainsi réalisées (fig. 1c: courbe en trait plein).

\subsubsection{Amélioration de la méthode}

Compte tenu de la dégradation progressive de la précision relative au stock, il est apparu que l'on pourrait s'affranchir de la connaissance du stock si l'on adoptait un point de vue "différentiel " consistant à comparer deux états successifs dé la cavité [9].

Supposons qu'aux instants $t_{1}$ et $t_{i+1}$, on ait effectué une thermométrie permettant d'accéder à la connaissance des couples (pression fond, température de fond): $\left(P_{1}, T_{1}\right)$ et $\left(P_{1+1}, T_{1+1}\right)$ et qu'entre ces deux instants, le volume libre ait varié dans un rapport: $\alpha_{1}=V_{1+1} / V_{1}$.

On trouve alors:

$$
V_{i}=\frac{\frac{P_{0}}{T_{0}}\left(S_{i+1}-S_{i}\right)}{\frac{\alpha_{i} P_{i+1}}{Z_{i+1} T_{i+1}}-\frac{P_{1}}{Z_{i} T_{i}}} .
$$

On constate que l'estimation du volume libre que l'on peut tirer de cette relation ne dépend que de la variation de stock, qui, par ailleurs, est grevée d'une incertitude relative beaucoup plus faible en général que le stock lui-même, si cette variation de stock est d'amplitude suffisante.

II faut cependant, pour rendre opératoire une telle méthode, pouvoir encadrer $\alpha_{1}$ dans une fourchette d'incertitude plausible; cela impose d'introduire des hypothèses exogènes vis-à-vis de la seule loi d'état du gaz. Le recours à un modèle de comportement mécanique de la cavité a permis d'estimer, en fonction de l'évolution de la pression entre $t_{i}$ et $t_{i+1}$, les bornes de l'intervalle d'incertitude de $\alpha_{i}$.

On peut également améliorer la méthode par l'emploi de la programmation linéaire. En effet, les contraintes auxquelles sont soumis les divers volumes et les stocks sont linéaires.

Pour i variant de 1 à n (nombre total de thermométries réalisées sur un puits), on peut écrire :

$$
\begin{aligned}
& \frac{p_{i}}{p_{0}} \cdot \frac{T_{0}}{T_{i}} \cdot \frac{1}{Z_{i}} \cdot V_{i}=S_{i} \\
& \sigma_{i} \leqslant S_{i+1}-S_{i} \leqslant \Sigma_{i} \\
& \alpha m_{1} \leqslant \frac{V_{i+1}}{V_{i}} \leqslant \alpha M_{1}
\end{aligned}
$$

la fonction économique à maximiser étant le volume lui-même.

\section{$\operatorname{Max} V_{1}$}

Ainsi, grâce à la programmation linéaire, on peut assurer la cohérence entre les diverses mesures, et réduire notablement l'incertitude qui entache une mesure élémentaire.

\subsection{Traçage à l'hydrogène}

C'est la détermination du stock qui grève lourdement l'estimation du volume d'une cavité par thermométrie. Au bout de 10 ans, l'incertitude relative sur le stock, et donc sur le volume de la cavité, peut atteindre $50 \%$.

Pour s'affranchir du comptage, la quantité de gaz en place peut être mesurée directement par traçage chimique.

Pour être valable, cette méthode nécessite que 3 hypothèses soient satisfaites :
- homogénéité du mélange traceur-gaz tracé en cavité : cette hypothèse a pu être vérifiée sur une petite cavité (Te 1) lors d'un déstockage important ( $75 \%$ du volume utile);

- représentativité de la mesure : on doit admettre que la concentration en gaz traceur dans le gaz soutiré, mesurée en surface, est représentative de la concentration en cavité,

- état du traceur dans la cavité : il faut supposer le traceur inerte vis-à-vis du gaz tracé, des divers autres fluides présents au fond de la cavité (saumure, fuel) et des parois de la cavité.

Pour des raisons à la fois techniques (inertie physico-chimique) et économique (coût du traceur), I'hydrogène a été adopté comme traceur [6].

Pratiquement, on commence par mesurer la teneur résiduelle $\tau_{1}$ en hydrogène du gaz en cavité, puis on injecte un volume $V_{H}$ d'hydrogène en cavité, suivi d'un volume $\Delta \mathrm{S}$ de gaz naturel afin d'assurer l'homogénéisation. De la teneur $\tau_{2}$ en hydrogène mesurée lors d'un soutirage élémentaire, on en déduit la valeur du stock.

$$
\left.\begin{array}{r}
\tau_{2} S_{2}=\tau_{1} S_{1}+V_{H} \\
S_{2}=S_{1}+\Delta S
\end{array}\right\} S_{2}=\frac{V_{H}-\tau_{1} \Delta S}{\tau_{2}-\tau_{1}}
$$

Cette méthode permet d'atteindre le stock en place avec une bonne précision $(2 \%)$, précision d'autant meilleure que l'écart entre les teneurs $\tau_{1}$ et $\tau_{2}$ est grand.

Ayant fait une thermométrie en fin d'injection de gaz naturel (stock $S_{2}$ ), on peut déterminer le volume libre par l'équation d'état.

On arrive ainsi à une précision sur le volume de l'ordre de $4 \%$.

\subsection{Résultats de 10 ans d'exploitation : Te 02}

Afin de confirmer les mesures de suivi du volume faites depuis 10 ans et surtout pour "voir» comment se déforme une cavité creusée dans le sel, la cavité Te 2 de Tersanne a été remise en saumure durant le deuxième semestre 1979 [12].

Cette cavité avait été lessivée de novembre 1968 à février 1970 .

Compte tenu des diverses mesures de volume effectuées à l'époque (mesure par écholog du volume total en saumure corrigé par la mesure de l'interface saumure-gaz et par l'estimation du lessivage complémentaire, comptage saumure corrigé de la compressibilité, comptage gaz couplé à la mesure de la température), on peut estimer que le volume initial libre au gaz de Te 2 était de $91000 \pm 2700 \mathrm{~m}^{3}$ et que le volume creusé initial, c'est-à-dire le volume libre au gaz augmenté du volume des " incompressibles ", était de $113000 \mathrm{~m}^{3} \pm 4000 \mathrm{~m}^{3}$ (le terme « incompressibles" désignant la saumure résiduelle et les insolubles foisonnés, voir fig. 2).

Le 10 juillet 1979, c'est-à-dire après 9 ans d'exploitation, une thermométrie associée à un traçage à I'hydrogène indiquait un volume libre au gaz de $58700 \pm 2000 \mathrm{~m}^{3}$.

La remise en saumure a eu lieu d'août à décembre 1979, la saumure injectée étant la plus concentrée possible (310 $\mathrm{g} / \mathrm{l}$ environ) afin d'éviter toute dissolution complémentaire.

Le 12 janvier 1980, l'écholog réalisé par la Société Prakla donnait un volume de $66600 \pm 3300 \mathrm{~m}^{3}$. Compte tenu d'une mesure d'interface saumure-gaz 
effectuée avant la remise en saumure, on peut admettre que le volume libre estimé par échométrie et interface était de $59800 \pm 4800 \mathrm{~m}^{3}$.

Cela permet donc d'affirmer, conformément aux mesures du suivi de volume effectuées depuis le début de l'exploitation, que le volume libre au gaz a diminué de $35 \%$ environ; cela correspond pour le volume creusé, compte tenu des insolubles et de la saumure résiduelle, à une diminution de $28 \%$ environ.

Le relevé échométrique (fig. 3) montre une déformation assez harmonieuse de la cavité. En admettant une déformation relative du rayon constante sur toute la hauteur, on peut évaluer que la remontée de l'interface saumure-gaz serait. de $3,5 \mathrm{~m}$ (au lieu de $5,8 \mathrm{~m}$ mesurés).

Cependant, les parois du puisard étant situées à une profondeur plus arande se sont probablement déformées davantage que le reste de la cavité.

Enfin, pour expliquer la remontée de l'interface saumure-gaz, on ne peut exclure la chute de blocs consécutive à la rupture des bancs en surplomb visibles sur le relevé de février 1970, le relevé 1980 ayant un aspect beaucoup plus lisse (on peut cependant également invoquer un fluage plus rapide pour le sel que pour l'anhydrite, ce qui donnerait aussi un aspect plus lisse au relevé).

Ainsi donc cette mesure exceptionnelle de contrôle a confirmé les mesures de contrôle de routine mises au point par le Département "Réservoirs Souterrains", Elle a permis également de montrer qu'une cavité saline pouvait se déformer dans des proportions importantes (domaine des grandes déformations) sans présenter de phénomène de rupture notable. Cette constatation légitime donc bien la recherche de paramètres rhéologiques décrivant le comportement du sel dans le cadre de la mécanique des milieux continus.

\section{Essais in situ}

Les mesures de surveillance de routine décrites précédemment permettent certes de déceler une tendance générale mais ne permettent malheureusement pas de caler finement un quelconque modèle de comportement du massif salifère. C'est pourquoi il est nécessaire de recourir à des essais in situ. Ces essais sont des moments privilégiés d'observation des cavités pendant lesquels il est possible de maîtriser la plupart des paramètres.

Les essais que I'on peut pratiquer sur une cavité saline sont variés, on peut les regrouper en 2 classes:

- pendant ou en fin de lessivage

- essai de compressibilité apparente

- essais en liquide;

- essais en gaz

- essais en cours de remplissage

- cavité pleine de gaz.

\subsection{Essais pendant ou en fin de lessivage}

\subsubsection{Essai de compressibilité apparente}

A l'issue du lessivage, avant de procéder à la mise en gaz de la cavité, on réalise divers essais, en particulier on mesure la compressibilité apparente de la cavité.

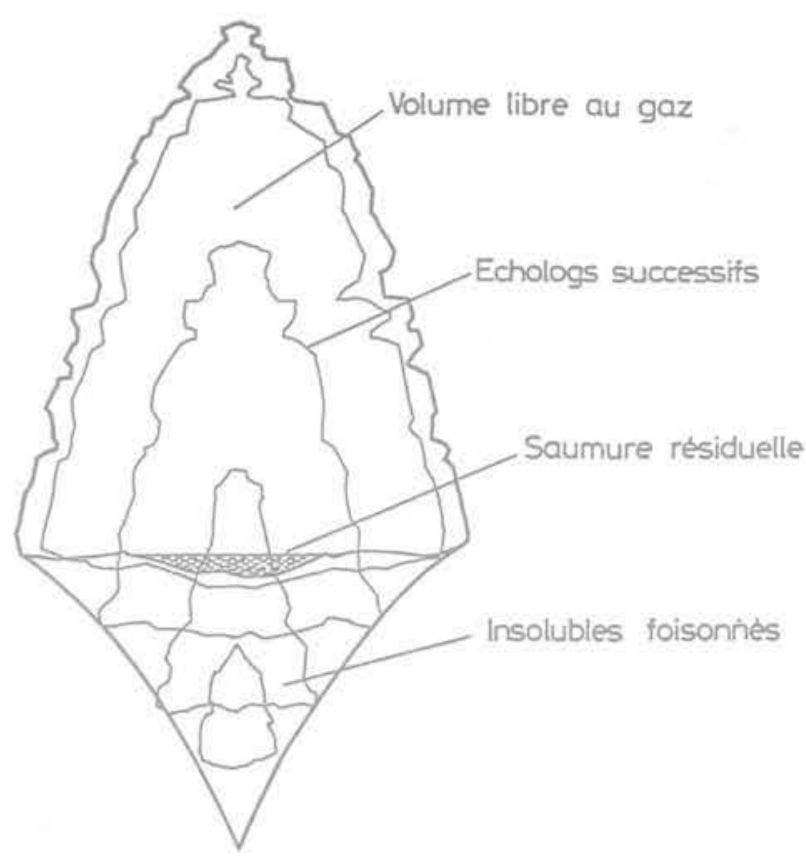

Fig. 2 Volume creusé et volume libre au gaz

L'essai consiste à injecter de l'eau douce par le tubing central et à observer la remontée de pression sur l'annulaire intermédiaire (fig. 4).

La variation de pression est de l'ordre de quelques dixièmes de Mégapascal. Le volume injecté dépend du volume de la cavité : il est de quelques dizaines de mètres cubes.

Cet essai est particulièrement intéressant puisqu'il permet d'accéder aux caractéristiques mécaniques élastiques des terrains.

En effet, vu la courte durée de l'essai (quelques heures) seule l'élasticité du massif est mobilisée. II est assez simple de voir que la compressibilité de l'ensemble " cavité + saumure " est la somme des compressibilités des terrains et de la saumure.

$$
\frac{1}{\mathrm{~V}} \frac{\mathrm{dV}}{\mathrm{dp}}=\beta_{\text {terrain }}+\beta_{\text {saumure }} \text {. }
$$

Remarque: Cette formule est valable en première approximation, en négligeant la présence des insolubles au fond de la cavité. L'erreur commise dans cette approximation est de l'ordre de $10 \%$.

Dans cette formule, $V$ représente le volume en saumure de la cavité déterminé par écholog, $\beta_{\text {saumure la }}$ compressibilité de la saumure, et $\beta_{\text {terrain }}$ la compressibilité des terrains.

En pratique, on trouve que $\frac{1}{V} \frac{d V}{d p}$ est à peu près constant, pour toutes les cavités et égal à 4 . $10^{-4} \mathrm{MPa}^{-1}$. Or la compressibilité de la saumure $\beta_{\text {saumure varie linéairement avec la concentration (à }}$ $310 \mathrm{~g} / \mathrm{l}, \beta_{\text {saumure }}$ vaut $2,7 \times 10^{-4} \mathrm{Mpa}^{-1}$ ), ce qui donne $\beta_{\text {terrain }}=1,3 \times 10^{-4} \mathrm{MPa}^{-1}$.

En ce qui concerne les terrains, le calcul en élasticité montre que: $\beta_{\text {terrain }}=f / \mu$ avec $f=0,75$ pour une sphère et $f=1$ pour un cylindre isolé dans un massif infini où $\mu=0,5 \times E /(1+v)$ désigne le module de cisaillement du matériau constituant le massif́.

Des calculs par éléments finis en élasticité pour une cavité piriforme montrent que $f=0,8$, coefficient par ailleurs très proche de celui de la sphère. 


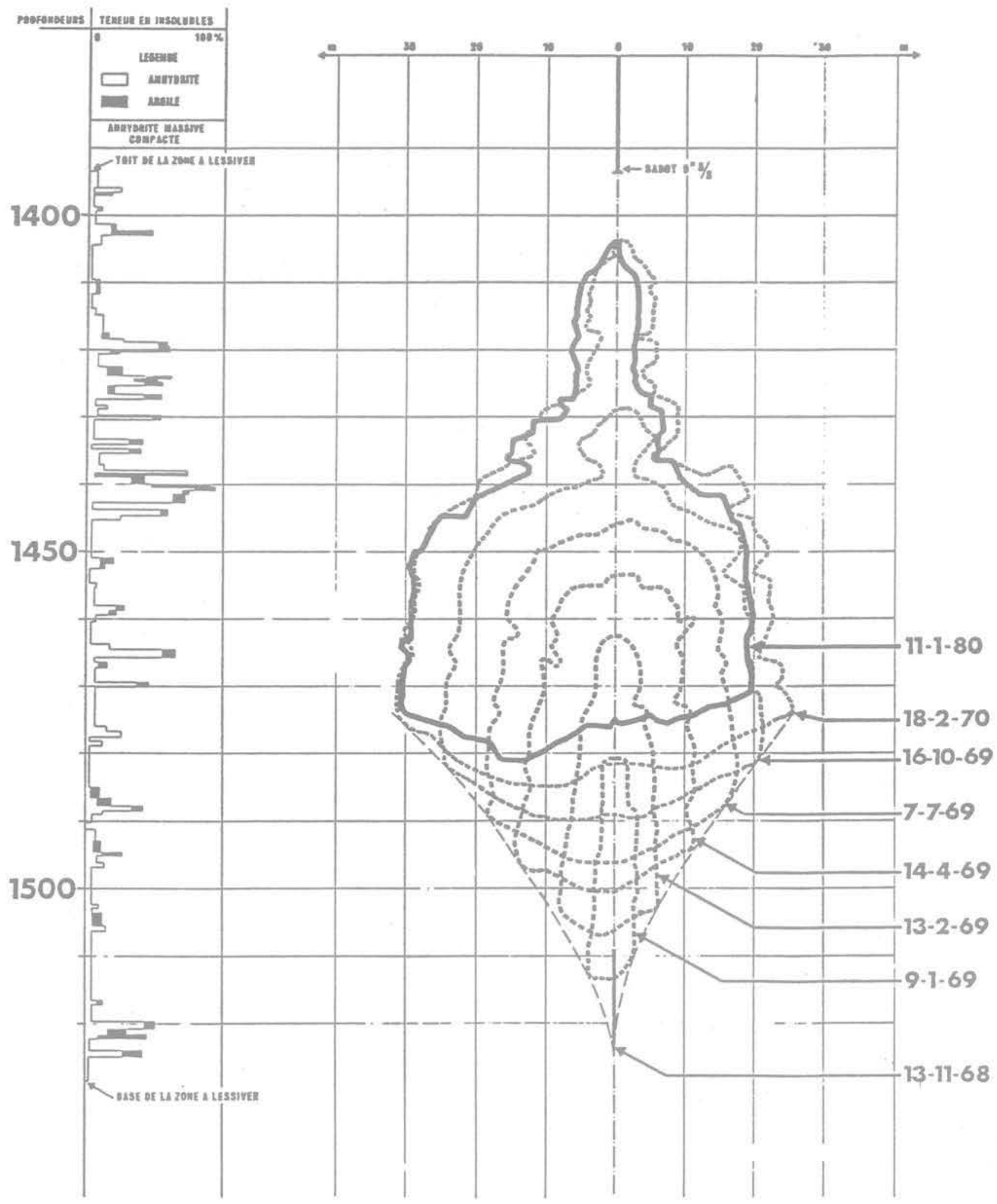

Fig. 3 Relevés échométriques successifs de la cavité Te 2

Ainsi à l'aide de toutes ces données, il est possible d'atteindre directement le module de cisaillement et moyennant l'hypothèse que le coefficient de Poisson $v$ vaut 0,25 on peut atteindre le modèle d'Young $E$ : $E$ de l'ordre de $15000 \mathrm{MPa}$.

L'incertitude sur la détermination de cette valeur est assez grande $(\approx 30 \%)$ : elle est due, essentiellement à l'incertitude sur le volume en saumure déterminé par échométrie.

\subsubsection{Essais en liquide}

L'essai décrit précédemment ne nous permet d'accéder qu'au comportement instantané et donc aux caractéristiques élastiques du massif supposé homogène et isotrope. Les essais en liquide, et en gaz, qui seront présentés ci-dessous permettent par leur durée d'atteindre le comportement différé du massif.

- Essai en saumure : la cavité est pleine de saumure, 


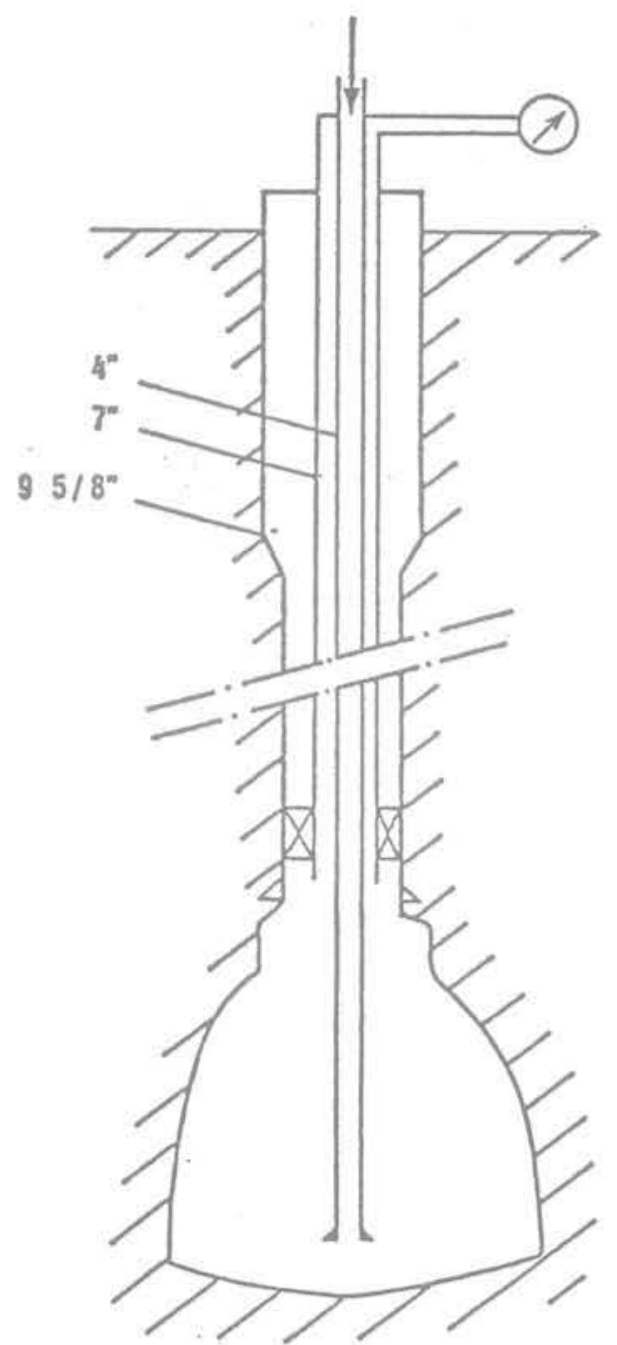

Fig. 4 Mesure de la compressibilité apparente d'une cavité

la tête de puits étant à la pression atmosphérique.

Un essai de ce type a été mené sur Te 02 à l'issue de sa remise en saumure.

La saumure étant saturée, cela correspondait à une pression en cavité de l'ordre de $17.5 \mathrm{MPa}$, soit un écart Q-P à la pression géostatique $Q$, de l'ordre de $16,5 \mathrm{MPa}$. Pendant toute la durée de l'essai (7 mois environ), de la saumure est constamment sortie du puits avec un débit journalier de $2,5 \mathrm{~m}^{3}$ /jour environ.

$\mathrm{Ce}$ débit de sortie est dû à deux phénomènes: la dilatation thermique de la saumure et le comportement non élastique du massif de sel. Pour apprécier le réchauffement de la saumure, des thermométries ont été effectuées régulièrement. Confirmées par des calculs analytiques, ces thermométries permettent d'estimer à $0,015^{\circ} \mathrm{C}$ par jour le réchauffement de la saumure. Compte tenu de la valeur du coefficient de dilatation volumique de la saumure $\alpha_{\mathrm{s}}=4,4 \cdot 10^{-4}{ }^{\circ} \mathrm{C}^{-1}$. cela correspond à un débit journalier de $0,5 \mathrm{~m}^{3} / \mathrm{j}$ environ. On en déduit donc que le débit de saumure dô au fluage du massif sous une pression intérieure de 17,5 $\mathrm{MPa}$ et pour une cavité ayant subi- une perte de volume de $30 \%$ est de l'ordre de $2 \mathrm{~m}^{3} / \mathrm{j}$.

La mise en œuvre de l'essai précédent est très simple puisqu'il suffit de disposer d'une cuve pour recueillir les 2 à $3 \mathrm{~m}^{3}$ sortis quotidiennement et d'effectuer régulièrement des thermométries en cavité. L'inconvénient est que l'on ne peut explorer qu'un palier de pression, celui correspondant à la pression d'une colonne de saumure saturée.
Pour explorer d'autres niveaux de pression, deux autres types d'essais ont été imaginés : en fuel et en saumure avec une pompe immergée.

- En fuel : la densité du fuel étant de $0,8 \mathrm{~kg} / \mathrm{dm}^{3}$, une colonne de fuel à Tersanne de la hauteur du casing correspond à une pression moyenne en cavité de l'ordre de $12 \mathrm{MPa}$. A ce niveau de pression, les modèles actuellement disponibles permettent de prévoir pour $\mathrm{Te} 2$ (volume en saumure de $66000 \mathrm{~m}^{3}$ ) un fluage de $30 \mathrm{~m}^{3} / \mathrm{j}$ environ, ce qui nécessiterait pour vérifier ce calcul sur une période d'un mois une quantité de fuel de $1000 \mathrm{~m}^{3}$ au minimum. Vu les problèmes pratiques posés (le lessivage d'une cavité ne nécessitant que $150 \mathrm{~m}^{3}$ de fuel au maximum), cet essaì ne peut être envisagé que sur de petites cavités (de l'ordre de $6000 \mathrm{~m}^{3}$ ). Dans ce cas, vu le fuel disponible et le débit prévisible $\left(4 \mathrm{~m}^{3} / \mathrm{j}\right)$, on pourrait effectuer un essai de 1 à 2 mois. Ce type d'essai sera tenté à Etrez au cours de l'année 1981.

- En saumure avec une pompe immergée. Cet essai consiste à immerger une pompe de faible diamètre $\left(\approx 4^{\prime \prime}\right)$ à une profondeur de l'ordre de $600 \mathrm{~m}$ par rapport au sol et à rabattre le niveau de la saumure dans le tubing. Grâce à un asservissement électrique et moyennant un fonctionnement intermittent de la pompe, il est possible de maintenir le niveau de la saumure entre deux bornes de façon à maintenir une pression sensiblement constante en cavité. Cet essai est encore à l'étude au Département "Réservoirs Souterrains $\nsim$ : il nécessitera de trouver une pompe de faible diamètre fournissant un petit débit sous une grande hauteur de refoulement.

L'avantage de ce type d'essai est de permettre d'explorer une large gamme de niveaux de pression. En attendant que soient définitivement arrêtées les conditions opératoires d'un tel essai, on a recours actuellement à des essais en gaz pour explorer des niveaux de pression différents des deux paliers précédents (fuel et saumure).

\subsection{Essais en gaz}

Leur mise en œuvre est de conception assez simple. On effectue l'essai à stock constant et on exécute régulièrement des thermométries et des mesures de la pression de tête. Un premier essai conduit selon cette méthode avait été mené sur Te 01 en fin 78 : il faisait apparaître une perte de volume de $5 \%$ au bout de 2 mois lorsque la pression en cavité était de $9 \mathrm{MPa}$ (écart à la pression géostatique $\mathrm{Q}-\mathrm{P} \approx 25 \mathrm{MPa}$ ) et une perte de volume de $2 \%$ au maximum en 2 mois pour une pression intérieure de $13,5 \mathrm{MPa}$ environ (écart $\mathrm{Q}-\mathrm{P}$ égal à $20,5 \mathrm{MPa}$ ). Malheureusement, vu les méthodes de mesure utilisées, cette variation de volume mesurée in situ était affectée d'une incertitude importante, de l'ordre de $4 \%$.

Pour remédier à cet inconvénient, l'essai peut être mené avec une cavité dont seul le sommet est mis en gaz. L'essai est mené également à stock constant : on relève quotidiennement la pression en tête et on effectue régulièrement des thermométries. L'avantage de cet essai est que les mouvements des parois de la cavité sont amplifiés au travers de la saumure et répercutés à la petite capacité de gaz située en sommet de cavité. La même méthode que dans le premier cas permet d'atteindre le taux de fluage mais avec une précision d'autant meilleure que la durée de l'essai est longue et que le volume occupé par le gaz est faible. Cet essai sera mené sur la cavité Te 2 au cours du $1^{\text {er }}$ semestre 1981. 
En conclusion, les essais que l'on peut pratiquer sur des cavités salines sont nombreux et variés. Tous, à l'exception de l'essai de la cavité totalement en gaz, présentent la particularité de devoir être faits soit en cours de lessivage (avec le fuel) soit en fin de lessivage (essai en saumure avec ou sans pompe immergée, essai avec une petite quantité de gaz en sommet de cavité). Seule l'observation d'une cavité laissée à stock constant pendant quelques mois permet d'atteindre le niveau de fluage à une pression donnée et ce à tout moment de la vie de la cavité. Malheureusement, cette détermination est grevée d'une incertitude importante, ce qui lui enlève une grande part de son intérêt.

\section{Modélisation}

\subsection{Démarche}

Les paragraphes précédents ont mis en évidence la richesse et la variété des observations faites ou possibles sur les cavités salines. Devant une telle diversité, deux comportements sont envisageables pour réaliser la synthèse indispensable :

Le premier consiste à adopter une démarche empirique, phénoménologique : on ne s'attache qu'aux grandeurs macroscopiques (volume, pression) que l'on essaie de relier par des formules ajustées sur les observations in situ.

En particulier, la variation relative de volume (VRV) d'une cavité est fréquemment exprimée par une formule :

$$
\text { VRV }=k(Q-P)^{\alpha} t^{\beta} \text { où } \begin{aligned}
& Q \text { désigne la pression géostatique } \\
& \mathrm{t} \text { la pression intérieure } \\
& \mathrm{t} \text { le temps }
\end{aligned}
$$

Cette approche est intéressante pour évaluer approximativement l'ordre de grandeur des variations de volume que peut subir une cavité en exploitation, à condition que la pression d'exploitation ne soit pas trop variable, ce qui n'est pas le cas pour les stockages de gaz exploités par détente. Elle n'est d'aucun intérêt pour l'implantation et le dimensionnement des cavités à créer.

La deuxième approche possible consiste en une approche locale : s'attachant aux grandeurs microscopiques (déformations, contraintes) et postulant des lois d'évolution de ces grandeurs microscopiques, on cherche à voir à quelles lois obéissent les grandeurs macroscopiques. Ayant constaté une analogie de comportement entre les cavités réelles simulées par éléments finis en élasticité et en élastoplasticité et une cavité sphérique isolée creusée dans un massif infini. on s'est attaché à rechercher quel était, pour une cavité sphérique, le comportement de la structure lorsqu'on se donne a priori le comportement du matériau, la forme sphérique se prêtant bien à des calculs analytiques au moins pour certains modèles de comportement.

Lorsque le matériau est supposé viscoélastique linéaire, régi par exemple par le modèle de Bürgers à plusieurs modèles de Kelvin, la variation relative de volume (VRV) peut être obtenue explicitement :

$$
\begin{aligned}
\text { VRV }=3\left(\frac{1+\nu}{2 E}\right) & (Q-P) \\
& +3 \sum_{k=1}^{n}\left(\frac{1+\nu_{k}}{2 \eta_{k}}\right) \int_{0}^{t}(Q-P(u)) e^{\frac{E k}{\eta k}(t-u)} d u
\end{aligned}
$$

où $\mathrm{E}$ et $v$ sont les paramètre élastiques; $E_{k}, \eta_{k}$ et $v_{k}$ les paramètres d'un des modèles de Kelvin.
Pour les modèles non linéaires, caractérisés par la présence d'un seuil, la démarche est moins aisée.

Les calculs de M. Salençon [1] ont d'abord permis d'apprécier la contraction quasi-statique d'une cavité à symétrie sphérique ou cylindrique dans un milieu élastoplastique parfait dans le cadre des grandes déformations. Puis, par les calculs de M. Tijani [5] il a été possible de simuler le comportement d'une cavité sphérique creusée dans un milieu obéissant au modèle de Bingham avec écrouissage et soumise à une dépression monotone.

Or les cavités de stockage de naturel exploitées par détente du gaz sont caractérisées par des variations de pression fréquentes et de grande amplitude; cela leur confère un caractère spécifique et les distingue des cavités de stockage d'hydrocarbures exploitées par balancement de saumure.

C'est pourquoi les calculs précédents ont du être généralisés: ils ont été repris dans l'hypothèse d'un chargement non monotone par M. Tijani [7] dans le cas de la plasticité parfaite pour une géométrie sphérique ou cylindrique et par MM. Berest et Nguyen Minh Duc[13] pour une cavité sphérique creusée dans un matériau obéissant au ômodèle de Bingham avec écrouissage.

Quand on adopte une démarche de calcul analytique ou semi-analytique de ce type, on se trouve rapidement dans l'impossibilité de prendre en compte des modèles rhéologiques assez généraux. Aussi a-t-on préféré recourir à des modèles maillés dont la structure modulaire se prête aisément à un changement de modèle de comportement et qui, étant fondé sur un calcul incrémental, sont particulièrement adaptés pour le calcul de sollicitations non monotones. C'est ainsi que pour rechercher un modèle rhéologique de comportement du sel rendant compte des déformations observées à Tersanne, on a utilisé le programme Cysiphe présenté par ailleurs par M. Tijani[15]. Ce programme permet actuellement de prendre en considération 2 modèles rhéologiques : le modèle dit du "C.M.R.-G.D.F.» constitué de 3 groupes de 3 modèles de Kelvin; chaque groupe ayant son seuil propre éventuellement nul, peut se représenter selon un schéma analogique monodimensionnel par la figure 5. C'est une généralisation des modèles rhéologiques habituels.

Le deuxième modèle considéré est le modèle de Lemaitre décrit par les équations ci-dessous :

$$
\dot{\varepsilon}^{p}=\dot{\xi} \frac{\tilde{\sigma}^{\prime}}{\left\|\tilde{\sigma}^{\prime}\right\|} \text { avec } \dot{\xi}=\left\|\dot{\varepsilon}^{p}\right\|=A \xi^{-n / m}\left\|\hat{\sigma}^{\prime}-2 C\right\|^{n}
$$

où $\bar{\sigma}^{\prime}$ est le déviateur de ō.

Ainsi que cela a été montré par M. Vouille[14], ce modèle nous est suggéré par le dépouillement des essais de laboratoire.

\subsection{Résultats}

Pour mener les premiers ajustements, un modèle très simple a été retenu. Sa description est donnée par la figure 6

$$
\mathrm{E}=20000 \mathrm{MPa} \text { et } y=0,25 .
$$

La cohésion $\mathrm{C}$ est de 1,5 à $2 \mathrm{MPa}$, c'est-à-dire l'ordre de grandeur trouvé au laboratoire, l'angle de frottement interne est nul.

Les constantes de viscosité $\eta_{1}$ et $\eta_{2}$ sont de quelques millions de MPa-jour. Les paramètres retenus sont les mêmes pour toutes les cavités. Seules changent les 


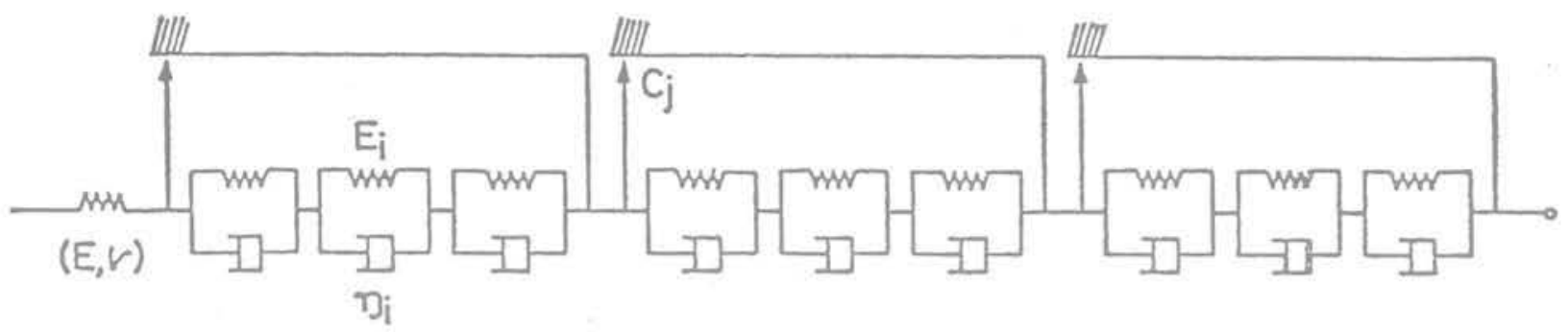

Fig. 5 Modèle du «CMR-GDF》

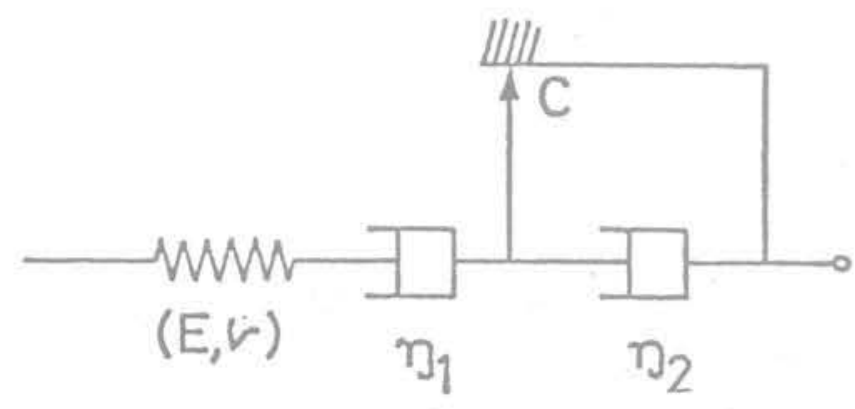

Fig. 6 Modèle retenu actuellement avec E module $d$ 'Young et $v$ coefficient de Poisson.
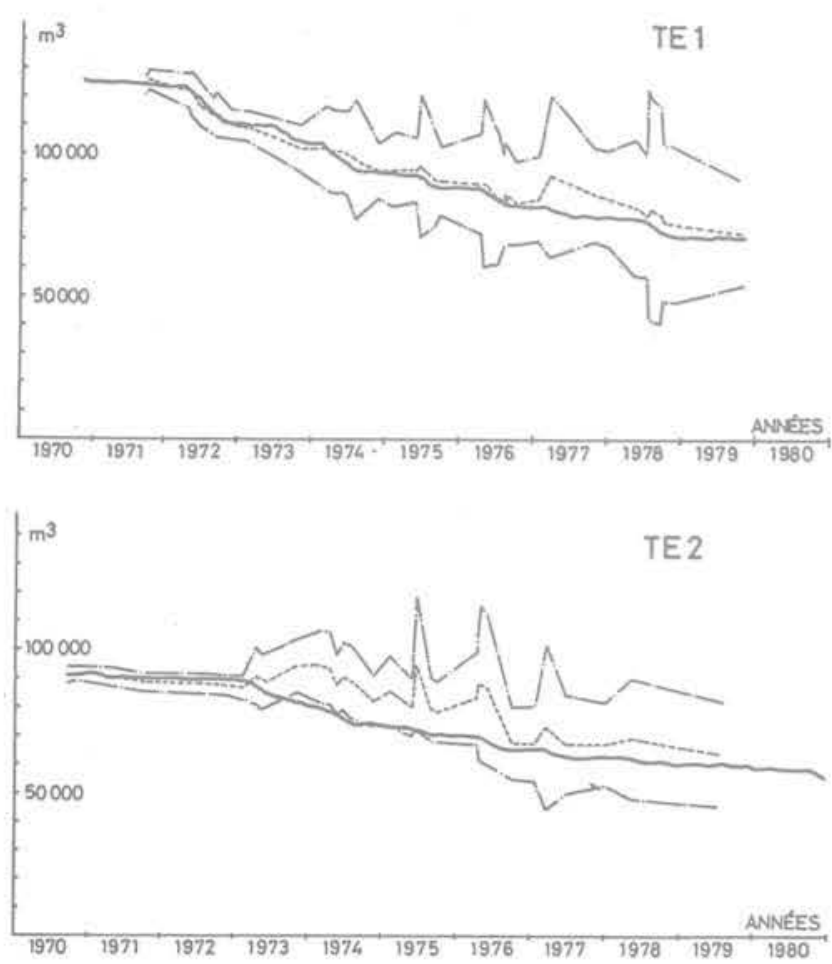

Depouillement par 1 thermometrie Incertitude sur le depouillement par 1 thermometrie Volume calculé par CYSIPHE

Fig. 7 Historique du volume des cavités Te 1 et Te 2 données géométriques c'est-à-dire la profondeur et donc la pression géostatique, ainsi que la taille de la cavité et donc aussi la durée du lessivage et le volume des incompressibles.

Grâce à ce modèle, il est possible de rendre compte correctement de l'ensemble des essais pratiqués sur les cavités de Tersanne, depuis 10 ans, l'écart entre le calcul et l'observation étant inférieur à l'erreur de mesure. La figure 7 montre le résultat de l'ajustement pour Te 1 et Te 2.

On notera certes au sujet de la cavité Te 2 pour la période 1973-1976 un écart notable entre la mesure et la prédiction du modèle. II faut supposer pour lever cette contradiction que les mesures du volume étaient erronées par suite vraisemblablement d'erreurs importantes sur le comptage. Il est en effet intuitivement peu acceptable que le volume d'une cavité suive une évolution aussi erratique que celle constatée au cours de cette période.

L'ajustement proprement dit ne pose pas de problème particulier, la difficulté essentielle réside dans l'incertitude qui entache tous les volumes in situ : en effet tous les volumes, et en particulier le volume initial, ne peuvent être obtenus avec une incertitude relative inférieure à $4 \%$. Cette remarque permet de mieux comprendre l'intérêt énorme des essais in situ en liquide ou partiellement en gaz qui donnent des résultats avec une précision bien supérieure.

\section{Conclusion}

Ainsi, grâce au travail des expérimentateurs qui, par les essais en laboratoire, et en particulier les essais de fluage, nous ont proposé certains modèles rhéologiques pour le sel, grâce au travail des théoriciens qui ont permis le calcul de structures obéissant à ces modèles, il a été possible de trouver un premier modèle de comportement du sel capable de synthétiser l'ensemble des observations faites sur les cavités. Vu la complexité du problème posé, le premier modèle retenu a dû être considérablement simplifié au point de n'avoir que 3 paramètres pour décrire le comportement différé; néanmoins même simplifié à ce point, il est capable de décrire n'importe quelle situation et en particulier prendre en compte l'histoire du matériau.

Naturellement, on devra à l'avenir vérifier que ce modèle permet d'intégrer les nouvelles expériences menées sur les cavités de Tersanne ou d'Etrez. Par ailleurs, d'autres types de comportements rhéologiques devront être explorés, afin de rechercher si des modèles différents permettent d'expliquer les mêmes phénomènes. II sera ainsi possible de tester la sensibilité des conclusions tirées non seulement à la valeur des paramètres d'un modèle donné mais aussi au modèle choisi lui-même. 
La première étape accomplie, à savoir la recherche d'un modèle de comportement des cavités de Tersanne supposées sphériques, il conviendra d'étudier le cas des cavités réelles grâce au programme d'éléments finis Viplef mis au point par M. Tijani. Cette étude comportera encore de nécessaires simplifications: géométrie axisymétrique, pas de pendage des couches, matériaux supposés homogènes et isotropes, schématisation des liaisons entre matériaux distincts. Elle permettra cependant de prendre en compte la géométrie réelle et l'hétérogénéité des terrains (bancs d'argile et d'anhydrite), le but de l'étude étant de valider le modèle rhéologique trouvé précédemment pour une cavité sphérique.

Ces applications immédiates étant effectuées, les recherches futures devront être menées dans quatre directions :

- Tout d'abord il s'agira de retrouver des règles pour l'espacement entre cavités ainsi que pour l'implantation dans la couche de sel. En effet les cavités de Tersanne ont été espacées de façon qu'à la pression minimale les zones plastiques de deux cavités voisines calculées dans l'hypothèse de l'élastoplasticité ne se rejoignent pas.

Comme l'a montré M. Vouille[14], les mesures récentes de laboratoire ont montré que le critère alors retenu était exagérément optimiste : cohésion de $3 \mathrm{MPa}$ et angle de frottement interne de $5^{\circ}$. A l'heure actuelle, cette règle n'est plus vérifiée compte tenu d'un angle de frottement interne nul et d'une cohésion de 1,5 à $2 \mathrm{MPa}$. II importe donc de retrouver qu'elle doit être la distance minimale entre deux cavités voisines pour éviter leur interaction ou du moins pour éviter toute instabilité notable.

- Sur le plan théorique, les programmes de calcul précédemment présentés par MM. Berest, Nguyen Minh Duc [13] et Tijani [15] devront être réécrits dans le cadre des grandes déformations. Les cavités Te 1 et Te 2 présentent des variations de volume de $30 \%$, ce qui est l'extrême limite de validité des solutions obtenues en petites déformations.

- Sur le plan expérimental, il conviendrait d'être capable d'appréhender l'hétérogénéité du massif : déjà, des méthodes, présentées par MM. Vouille [14] et Bergues, ont permis de mener un certain nombre d'essais sur la même éprouvette. Afin d'étudier plus en détail le problème de la dispersion et d'être capable de comparer entre eux des résultats obtenus sur des échantillons différents par des méthodes différentes, il importerait de savoir décrire et quantifier (analyse minéralogique, taille des grains, description des joints de grain, etc.) les matériaux étudiés. Cela permettrait également sans doute de transposer à des sites nouveaux un savoir et une connaissance du massif salifère de Tersanne particulièrement riche.

- Enfin, bien que notre expérience nous ait montré que le problème ne se posait pas de façon aiguë, il importerait d'explorer le domaine de la rupture du sel (et éventuellement d'autres matériaux tels que l'anhydrite). Cette étude devrait se faire à la fois sur le plan expérimental (la rupture a-t-elle lieu pour une contrainte excessive ou une déformation excessive ou plutôt, comme le suggère M. Tijani, pour une énergie de déformation excessive?) et sur le plan théorique : comment considérer les éléments violant le critère de rupture? Participent-ils toujours au soutènement? L'utilisation de la théorie du post pic, largement développé par ailleurs par MM. Berest et Nguyen Minh Duc pourrait, à notre avis, représenter une schématisa- tion intéressante de l'endommagement du sel et décrire sa perte de cohésion lorsqu'il est sollicité de façon excessive.

\section{Références bibliographiques}

[1] Salençon (1969), Contraction quasi-statique d'une cavité à symétrie sphérique ou cylindrique dans un milieu élastoplastique. Annales des Ponts et Chaussées (juillet-aout 1969).

[2] Pottier et Leguillette (1971). Le stockage souterrain de gaz naturel dans le sel. Réalisation de Tersanne. Congrès A.T.G. 1971.

[3] Roux et Pottier (1974). Communication au Congrès U.I.G. 1974 à Nice.

[4] Pottier et Dussaud (1975). Problème de métrologie dans la surveillance des cavités de stockage dans le sel. Congrès A. T. G. 1975.

[5] Tijani (1975). Rhéologie du sel gemme. Rapport interne du Centre de Mécanique des Roches de Fontainebleau.

[6] Dussaud et Coquand (1977). Détermination du stock en place dans une cavité de stockage de gaz naturel par une méthode de traçage chimique à I'hydrogène. Congrès A. T. G. 1977.

[7] Tijani (1977). Influence des cycles d'injection, soutirage sur la variation du volume d'une cavité de stockage et sur l'extension de la zone plastique. Rapport interne du Centre de Mécanique des Roches de Fontainebleau.

[8] Lasneret et Vernet (1978). Le stockage souterrain d'Etrez. Aménagements de lessivage et premiers résultats. Congrès A.T.G. 1978.

[9] Boucher, Boucly et Carrière (1979). Problèmes posés par le comportement mécanique du sel dans la création et le contrôle des cavités de stockage de gaz naturel. Congrès U.I.G. 1979 à Toronto.

[10] Les stockages souterrains de gaz en cavités salines : dix années d'études théoriques et d'expérimentation. Colloque B.R. G. M. à Lyon. Mars 1979.

[11] Fine et al (1979). Détermination expérimentale de quelques paramètres élastoviscoplastiques de roches. Application aux cavités de stockage de gaz en couches salines profondes. $4^{\circ}$ Congrès international de Mécanique des Roches. Montreux 1979.

[12] Boucly et Legreneur (1980). Hydrocarbon storage in cavities leached out of salt formation. Rockstore 80. Stockholm 1980.

[13] Berest et Nguyen Minh Duc. Modélisation d'une cavité de stockage de gaz dans le sel considéré comme matériau élastoviscoplastique. Solution explicite. Revue Française de Géotechnique n 16. Août 1981.

[14] Vouille. Détermination expérimentale du comportement rhéologique du sel de Tersanne. Revue Française de Géotechnique. (A paraître.).

[15] Tijani. Modélisation numérique du comportement des cavités de stockage en couches salines profondes. Revue Française de Géotechnique. (A paraître.) 
